\title{
Design concept: A displaced anecdote in the landscape architectural design process
}

\begin{abstract}
In the landscape architecture design process, "concept" is important for providing uniqueness to a design. However, many landscape designers today do not rely on a well-defined concept, thus obstructing the development of their designs. This study aimed to identify the problems that Malaysian landscape designers face in developing a strong design concept. To address this issue, in-depth and focus group interviews were conducted by guiding the participants through a conceptual thinking procedure in which eighteen participants took part. The respondents were chosen among Malaysian professional landscape designers through purposeful sampling. Results indicated that most Malaysian landscape designers neglect the post- design evaluation stage, which obstructs the development of a strong concept for subsequent landscape designs. The results aim to contribute to a better understanding of the importance of design concept and the factors that contribute to its development among Malaysian landscape designers.
\end{abstract}

Keyword: Concept, Design Process, Landscape Design 